\section{Cureus}

Received 03/25/2019

Review began 03/27/2019

Review ended 03/27/2019

Published 04/01/2019

\section{(c) Copyright 2019}

Maheshwari et al. This is an open access article distributed under the terms of the Creative Commons Attribution License CC-BY 3.0., which permits unrestricted use, distribution, and reproduction in any medium, provided the original author and source are credited.

\title{
Impact of Poor Sleep Quality on the Academic Performance of Medical Students
}

\author{
Ganpat Maheshwari ${ }^{1}$, Faizan Shaukat ${ }^{2}$ \\ 1. Internal Medicine, Jinnah Sindh Medical University, Karachi, PAK 2. Internal Medicine, Jinnah Post \\ Graduate Medical Center, Karachi, PAK
}

Corresponding author: Faizan Shaukat, faizan.shaukat89@gmail.com

\section{Abstract}

Introduction: Adequate sleep has a crucial role in enhancing cognitive skills especially memory retention. Poor night time sleep quality and the consequent daytime sleepiness affect physical and cognitive health of students and their academic performance. The aim of this study is to find whether or not poor academic performance is a consequence of poor sleep quality among Pakistani medical students.

Methods: It was an observational, cross-sectional study conducted with undergraduate medical students. Pittsburgh Sleep Quality Index (PSQI) was used to assess the sleep quality. Academic performance was assessed by mean grade point average (GPA) of the students. The data were analyzed using the Statistical Package for the Social Sciences (SPSS) 22.0 (IBM Corp., Armonk, NY, USA).

Results: There were 512 (64.24\%) students with global PSQI score $\geqslant 5$ indicating poor sleep quality. The mean GPA of poor sleepers was $2.92 \pm 1.09$ which was significantly lower than that of good sleepers $(\mathrm{p}<0.0001)$. In the group of students who scored lower GPA $(2.0-2.7), 28.2 \%$ had very bad subjective sleep quality, $29.05 \%$ had sleep latency of $16-30 \mathrm{~min}, 29.4 \%$ had sleep duration of $<5-7 \mathrm{~h}, 27.8 \%$ had sleep efficiency of $<85 \%$, and $37.7 \%$ experienced daytime dysfunction almost every day.

Conclusion: Medical students of Pakistan have poor sleep quality which has a negative impact on their academic performance. Adequate sleep is essential to refresh the students every day and help them in learning and memory processing. Medical students and their facilitators should comprehend the negative effects of sleep deprivation on student academics and should take adequate measures to improve the sleep quality of students.

Categories: Medical Education, Psychology, Miscellaneous

Keywords: pittsburgh sleep quality index, sleep quality, medical students, academic performance, grade point average, poor sleeper, daytime dysfunction

\section{Introduction}

Adequate sleep of high quality and optimum duration facilitates memory processing and learning. It helps maintain concentration, executive cognitive functions, sensorimotor integration, and memory processing [1]. Sleep patterns and habits are different for different individuals depending on their age, occupational demands, social engagements, psychiatric and somatic conditions, and also individual physiological characteristics [2-3].

Young and old adults are recommended to sleep for seven to nine hours every night [4]. 
However, recent literature predominantly shows how most of the young adults are sleeping for less than the recommended duration [5-6]. The relationship of sleep inadequacy with stress is that of a chain reaction. Studies have established that sleep disturbances are, at times, caused by psychosocial stressors and also that psychosocial stressors culminate in sleep inadequacy [23, 5-6]. Disturbed and inadequate sleep leads to judgement impairment, agitation, irritability, and inability to process information in the short term, and in the long term, it can contribute to cardiometabolic disorders and even increased mortality [7].

One of the high-risk groups, established in the literature, for being affected by disorders of sleep are students $[2-3,5]$. A high propensity has been seen in young students attending medical schools [6-7]. In medical students, various studies have been conducted to assess sleep disturbances and their results vary based on their years of education and their geodemographic locations. In Chinese medical students, approximately $90 \%$ or more reported in-class daytime sleepiness [8] and the percentage was $35.5 \%$ for Malaysian students which was more common in clinical students [9]. In Hong Kong, medical students had a mean night-time sleep duration of $6.6 \pm 1.2 \mathrm{~h} ; 70 \%$ of them complained of sleep deprivation [10]. Poor sleep quality has been reported in $16 \%$ of Malaysian medical students [9], 40.6\% of Iranian medical students-with highest prevalence in their interns [11], 62.6\% of Indian students [12], and 77\% of Pakistani medical students [13].

Literature has extensively reported high prevalence of poor sleep quality among medical students. However, not many researchers have studied the consequences of inadequate sleep quality. It has been proposed that sleep deprivation causes depression, agitation, apathy, and poor academic performance in students [14]. Reduced total sleeping hours have been associated with declining academic performance [15].

As stated earlier, studies have reported the frequency of poor sleepers among Pakistani medical students and its association with academic stress [13]; studies have not yet reported the impact of proper sleep on the academic performance of these students. The aim of this study is to find any correlation between poor sleepers and their grade point average (GPA).

\section{Materials And Methods}

It was an observational, cross-sectional study conducted with undergraduate students in public medical university of Karachi, Pakistan. The study duration was from 1st January till 15th March 2019. Students of both genders, and all five years of education were included. With approximately 350 students in each batch, all 1750 MBBS students were invited, out of which 980 students responded. There were 183 incomplete responses which were excluded. Remaining 797 responses were included in the study (response rate: $45.54 \%$ ).

A structured questionnaire, consisting of two sections, was distributed. The first section comprised demographic information including gender, age, and GPA of every semester. The second part comprised Pittsburgh Sleep Quality Index (PSQI). PSQI is an efficient measure of the quality and pattern of sleep. It assesses sleep quality on seven components-subjective sleep quality, sleep latency, sleep duration, habitual sleep efficiency, sleep disturbances, use of sleeping medication, and daytime dysfunction. Its scores range from minimum zero to maximum 21. The combined score of all seven components is termed as "global score of PSQI." Global PSQI score $\geqslant 5$ signifies "poor sleep quality.” The PSQI has internal consistency and a reliability coefficient (Cronbach's alpha) of 0.83 for its seven components [16].

The SPSS for Windows, version 22.0 (IBM Corp., Armonk, NY, USA) was used to enter and analyze the data. Demographic data were categorized to calculate frequencies and percentages. All components of PSQI were categorized as mentioned by Smyth [16]. Mean GPA was calculated for every student. GPA was recorded as a numerical variable. It was then transformed 


\section{Cureus}

to categorical variable by sub dividing into three groups $(<2.7,2.71-3.40$, and $>3.41)$. All scores of PSQI were compared against GPA. Chi square was calculated for correlation between PSQI score and GPA. A p value of $\leqslant 0.05$ was taken as significant.

\section{Results}

Out of the 791 students, 531 (66.62\%) were females and 266 (33.37\%) were males. Mean GPA was $3.08 \pm 1.12$ for the entire study sample. There were 241 (30.23\%) students with GPA less than 2.71, 312 (39.14\%) students with GPA 2.71-3.40 and 244 (30.61\%) with GPA 3.41-4.00.

Poor sleep quality was witnessed in 512 (64.24\%) students. Their mean GPA was $2.92 \pm 1.09$. There were 285 (35.76\%) students who were not poor sleepers and their PSQI score was $<5$. Their mean GPA was $3.31 \pm 1.49$. Poor sleepers had significantly lower mean GPA $(\mathrm{p}<0.0001)$.

All seven components of PSQI were compared with the average GPA of the students. Most of the students with lower GPA (2.0-2.7) had very bad subjective sleep quality (28.2\%), sleep latency of 16-30 min (29.05\%), sleep duration of <5-7 h (29.4\%), and sleep efficiency of 75\%-84\% (27.8\%). Most of these students experienced sleep disturbances once or twice a week (29.8\%), had not used sleep medication in the past one month (87.14\%), but experienced daytime dysfunction almost every day (37.7\%). The comparison of their sleep quality parameters to groups with higher GPAs is shown in Table 1.

\section{Components of PSQI}

GPA 2.0 to $2.7(n=241)$

GPA 2.71 to $3.40(n=312)$

GPA 3.41 to $4.00(n=244)$

\section{Subjective sleep quality}

Very good

Fairly good

Fairly bad

Very bad

Sleep latency

$\leq 15 \min$

$16-30 \mathrm{~min}$

$31-60 \mathrm{~min}$

$>60$ min

Sleep duration

$>7 \mathrm{~h}$

6-7 h

5-6 h

$<5 \mathrm{~h}$

Habitual sleep efficiency

$>85 \%$
$31(12.86 \%)$

$71(29.46 \%)$

$68(28.22 \%)$

$71(29.46 \%)$

$52(21.58 \%)$

$74(19.50 \%)$

$47(19.50 \%)$

$68(28.22 \%)$

$56(23.24 \%)$

$70(29.05 \%)$

$59(24.48 \%)$

$56(23.24 \%)$

$87(27.88 \%)$

$42(13.46 \%)$

$86(27.56 \%)$

107 (34.29\%)

77 (24.68\%)

53 (16.99\%)
$61(33.20)$

$81(33.20 \%)$

57 (23.36\%)

45 (18.44\%)

$71(29.10 \%)$

$65(26.64 \%)$

57 (23.36\%)

$51(20.90 \%)$

$36(14.75 \%)$

$90(36.89 \%)$

67 (27.46\%)

$51(20.90 \%)$ 


\section{Cureus}

\begin{tabular}{|c|c|c|c|}
\hline $75 \%-84 \%$ & $67(27.80 \%)$ & $102(32.69 \%)$ & $81(33.20 \%)$ \\
\hline $65 \%-74 \%$ & $61(25.31 \%)$ & $74(23.72 \%)$ & $74(30.33 \%)$ \\
\hline$<65 \%$ & $79(32.78 \%)$ & $83(26.60 \%)$ & $50(20.49 \%)$ \\
\hline \multicolumn{4}{|l|}{ Sleep disturbances } \\
\hline Not during the past month & $50(20.75 \%)$ & $91(29.17 \%)$ & $59(24.18 \%)$ \\
\hline Less than once a week & 49 (20.33\%) & $88(28.81 \%)$ & $83(34.01 \%)$ \\
\hline Once or twice a week & $72(29.88 \%)$ & $60(19.23 \%)$ & $59(24.18 \%)$ \\
\hline Three or more times a week & $70(29.05 \%)$ & $73(23.40 \%)$ & $43(17.62 \%)$ \\
\hline \multicolumn{4}{|l|}{ Use of sleep medication } \\
\hline Not during the past month & $210(87.14 \%)$ & $271(86.86 \%)$ & $231(94.67 \%)$ \\
\hline Less than once a week & $19(7.88 \%)$ & $28(8.97 \%)$ & $11(4.51 \%)$ \\
\hline Once or twice a week & $10(4.15 \%)$ & $12(3.85 \%)$ & $2(0.82 \%)$ \\
\hline Three or more times a week & $2(0.83 \%)$ & $1(0.32 \%)$ & $0(0.00 \%)$ \\
\hline \multicolumn{4}{|l|}{ Daytime dysfunction } \\
\hline 1-2 days & $53(21.99 \%)$ & $81(25.96 \%)$ & $72(29.51 \%)$ \\
\hline 3-4 days & $50(20.75 \%)$ & $76(24.36 \%)$ & $71(29.10 \%)$ \\
\hline 5-6 days & $47(19.50 \%)$ & $73(23.40 \%)$ & $58(23.77 \%)$ \\
\hline Everyday & $91(37.76 \%)$ & $82(26.28 \%)$ & $43(17.62 \%)$ \\
\hline
\end{tabular}

TABLE 1: Sleep quality as assessed by PSQI in medical students correlated with their mean GPA ( $n=791)$.

PSQI, Pittsburgh Sleep Quality Index; GPA, grade point average.

\section{Discussion}

Adequate and efficient sleep plays a crucial role in learning and memory. It is important for students to sleep well in order to perform well in academics. The results of this study are very alarming. Most students with lower average GPA reported very bad subjective sleep quality, had a sleep latency of $16-30 \mathrm{~min}$, sleep duration of $<5-7 \mathrm{~h}$, sleep efficiency of $75 \%-84 \%$, and experienced daytime dysfunction almost every day.

The study provides robust evidence regarding the association of sleep disturbances with declining pattern in mean GPA scores. However, the study has its limitations too. It has not taken into account or excluded other factors such as exam stress, exam difficulty, etc. that can contribute to poor academic performance. Also, it was conducted among students of only one medical college and cannot be generalized for the entire population. This is a cross-sectional 
study which only shows an association and, in no manner, has determined causality.

Even with our best efforts, we could not find any local data published. However, there is substantial evidence from other countries regarding the delirious effects of sleep deprivation on students' academic performance. Among Saudi medical students, sleep disturbances including insomnia, frequent awakenings, and falling asleep after $30 \mathrm{~min}$ of going to bed were common [6]. Similarly, Indian students who slept for shorter duration reported lower GPAs and poor memory and concentration [17].

Philips in 2017 in his research stated that for every 10-point increase in sleep regularity index, a 0.10 increase in GPA has been observed. Furthermore, negative linear correlations have been observed between GPA and sleep onset time, wake time, and mid-sleep time [18]. In Sudanese medical students, there were significant differences in the overall sleep quality, subjective sleep rating, bedtime later than midnight, sleep latency, and daytime dysfunction between students who scored an " $\mathrm{A}$ " in the exams and those who scored a "C." Their " $\mathrm{A}$ " scoring group had a higher mean sleep duration than the "C" scoring group [19]. In Iran, 85\% of the medical students who scored a GPA of 2.99 or less were poor sleepers on PSQI [20]. If looked together, the results suggest a strong negative relationship between sleep quality and academic performance of the medical students.

\section{Conclusions}

Medical students are continuously under high academic stress and pressure. Adequate sleep is essential to refresh them every day and help in learning and memory processing. Sleep disturbances are common in medical students and worsen their academic performance. Medical students and their facilitators should comprehend the negative effects of sleep deprivation on student academics and should take adequate measures to improve the sleep quality of students.

\section{Additional Information \\ Disclosures}

Human subjects: Consent was obtained by all participants in this study. Jinnah Sindh Medical University Ethical Review Board issued approval 18/233. Animal subjects: All authors have confirmed that this study did not involve animal subjects or tissue. Conflicts of interest: In compliance with the ICMJE uniform disclosure form, all authors declare the following:

Payment/services info: All authors have declared that no financial support was received from any organization for the submitted work. Financial relationships: All authors have declared that they have no financial relationships at present or within the previous three years with any organizations that might have an interest in the submitted work. Other relationships: All authors have declared that there are no other relationships or activities that could appear to have influenced the submitted work.

\section{References}

1. Vyazovskiy VV: Sleep, recovery, and metaregulation: explaining the benefits of sleep . Nat Sci Sleep. 2015, 7:171-184. 10.2147/NSS.S54036

2. Lemma S, Gelaye B, Berhane Y, Worku A, Williams MA: Sleep quality and its psychological correlates among university students in Ethiopia: a cross-sectional study. BMC Psychiatry. 2012, 12:237. 10.1186/1471-244X-12-237

3. Augner C: Associations of subjective sleep quality with depression score, anxiety, physical symptoms and sleep onset latency in students. Cent Eur J Public Health. 2011, 19:115-117.

4. National Sleep Foundation Recommends New Sleep Times . (2015). Accessed: March 7, 2019: https://www.sleepfoundation.org/press-release/national-sleep-foundation-recommendsnew-sleep-times. 
5. Quick V, Byrd-Bredbenner C, Shoff S, et al.: Relationships of sleep duration with weightrelated behaviors of US college students. Behav Sleep Med. 2016, 14:565-580. 10.1080/15402002.2015.1065411

6. Alsaggaf MA, Wali SO, Merdad RA, Merdad LA: Sleep quantity, quality, and insomnia symptoms of medical students during clinical years: relationship with stress and academic performance. Saudi Med J. 2016, 37:173-182. 10.15537/smj.2016.2.14288

7. Yazdi Z, Loukzadeh Z, Moghaddam P, Jalilolghadr S: Sleep hygiene practices and their relation to sleep quality in medical students of Qazvin University of Medical Sciences. J Caring Sci. 2016, 5:153-160.

8. Lu J, Fang GE, Shen SJ, Wang Y, Sun Q: A questionnaire survey on sleeping in class phenomenon among Chinese medical undergraduates. Med Teach. 2011, 33:508.

9. Zailinawati AH, Teng CL, Chung YC, Teow TL, Lee PN, Jagmohni KS: Daytime sleepiness and sleep quality among Malaysian medical students. Med J Malaysia. 2009, 64:108-110.

10. Huen LL, Chan TW, Wai-Man MY, Wing YK: Do medical students in Hong Kong have enough sleep?. Sleep Biol Rhythms. 2007, 5:226-230. 10.1111/j.1479-8425.2007.00278.x

11. Ghoreishi A, Aghajani AH: Sleep quality in Zanjan university medical students. Tehran Univ Med J. 2008, 66:61-67.

12. Shad R, Thawani R, Goel A: Burnout and sleep quality: a cross-sectional questionnaire-based study of medical and non-medical students in India. Cureus. 2015, 7:361. 10.7759/cureus.361

13. Waqas A, Khan S, Sharif W, Khalid U, Ali A: Association of academic stress with sleeping difficulties in medical students of a Pakistani medical school: a cross sectional survey. Peer J. 2015, 3:840. 10.7717/peerj.840

14. Alalageri KM, Sobagaih RT: A cross sectional study to determine the sleep pattern and impact of sleep deprivation on the health and academics of medical students of BMCRI Bengaluru. Int J Community Med Public Health. 2017, 4:3731-3734. 10.18203/2394-6040.ijcmph20174241

15. Abdulghani HM, Alrowais NA, Bin-Saad NS, Al-Subaie NM, Haji AM, Alhaqwi AI: Sleep disorder among medical students: relationship to their academic performance. Med Teach. 2012, 34:37-41. 10.3109/0142159X.2012.656749

16. Smyth C: The Pittsburgh Sleep Quality Index (PSQI) . J Gerontol Nurs. 1999, 25:10. 10.3928/0098-9134-19991201-10

17. Rose S, Ramanan S: Effect of sleep deprivation on the academic performance and cognitive functions among the college students: a cross sectional study. J Chalmeda Anandrao Inst Med Sci. 2017, 14:51-56.

18. Phillips AJ, Clerx WM, O’Brien CS, et al.: Irregular sleep/wake patterns are associated with poorer academic performance and delayed circadian and sleep/wake timing. Sci Rep. 2017, 7:3216. 10.1038/s41598-017-03171-4

19. Mirghani HO, Mohammed OS, Almurtadha YM, Ahmed MS: Good sleep quality is associated with better academic performance among Sudanese medical students. BMC Res Notes. 2015, 8:706. 10.1186/s13104-015-1712-9

20. Rasekhi S, Pour Ashouri F, Pirouzan A: Effects of sleep quality on the academic performance of undergraduate medical students. Health Scope. 2016, 5:31641. 10.17795/jhealthscope31641 